\title{
Open Access Journal of Ophthalmology
}

\section{CNS Whipple's Disease Heralded by Retinal Vasculitis}

\author{
Thanh Le ${ }^{1 *}$, Ramirez $A^{2}$, 0’Connor $P^{2}$, Youssef $0^{1}$, Agange $N^{2}$, Dipti \\ Singh $\mathrm{OD}^{1}$ and Wentworth $\mathrm{G}^{1}$ \\ ${ }^{1}$ Chief ophthalmologist at South Texas Veterans Health Care System, USA \\ ${ }^{2}$ Department of Ophthalmology, University of Texas Health Science Center, USA \\ *Corresponding author: Thanh Le, VA Eye Clinic 8410 Datapoint Drive San Antonio, \\ TX 78256, USA, Tel: 361-876-2071; E-mail: thanh.le3@va.gov
}

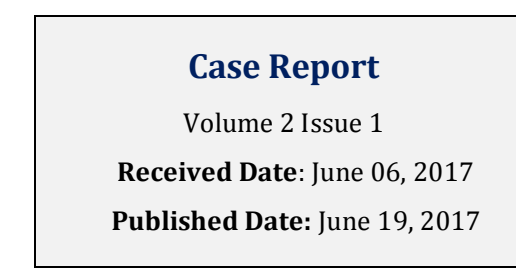

\section{Abstract}

Background: Whipple's disease is a rare, chronic, multi-organ, bacterial infection. The most common presenting manifestation of Whipple's disease is gastrointestinal symptoms such as abdominal pain, weight loss, diarrhea, and migratory non-deforming sero negative polyarthralgias. Although neuro-ophthalmologic symptoms are common in CNS Whipple's disease, uveitis as a presenting sign is rare.

Case Report: A 36-year-old black male presented with the initial complaint of left eye vision loss but left against medical advice, only to return nine weeks later with additional symptoms of right eye visual field loss. Ocular examination revealed panuveitis, vasculitis, optic nerve atrophy left eye, and right homonymous hemianopsia of both eyes. Extensive laboratory testings were all negative. A MRI imaging showed large enhancing lesions in the thalamus, temporal and parietal lobes. A brain biopsy revealed periodic acid-Schiff reagent (PAS)-positive intracytoplasmic organisms within multiple macrophages, consistent with Whipple's disease. After two months of antibiotic therapy, the patient's symptoms and MRI findings were markedly improved.

Conclusion: Although rare, Whipple's disease should be considered in any unexplained chronic uveitis especially if accompanied by CNS, gastrointestinal or migratory polyarthralgia symptoms.

Keywords: Whipple’s Disease; Panuveitis; Vasculitis; Opticatrophy; Visual field loss; Tropheryma whipplei

\section{Introduction}

Whipple's disease, once thought to be an inflammatory disease of the gastrointestinal tract of unknown origin, was first reported in 1895 by Allchin and Hebb but not until 1907 was it recognized as a unique "intestinal lipodystrophy" disease by the pathologist George Hoyt Whipple which later bared his name. The first case of ocular Whipple's disease was reported in 1949. Although the signs and symptoms of Whipple's disease were well known for years the causative bacterium was only successfully identified as Tropheryma whipplei in 1992. 


\section{Open Access Journal of Ophthalmology}

T. whipplei is an actinomycete organism that often appears in environments like soil and animal's wastes. The disease occurs predominantly in middle age Caucasian males and is extremely rare with approximately 1000 reported cases to date [1]. Rickman reported one case of Whipple's disease of the eye without any apparent CNS or gastrointestinal involvement [2]. Most clinical eye manifestations are nonspecific including uveitis, retinal hemorrhage,, papilledema, cornea ulcer, chemosis, glaucoma, epiphora, and optic atrophy, thus making a proper diagnosis is quite difficult leading to delays in treatment and poor prognosis. The purpose of this report is to describe a case of ophthalmic signs and symptoms that can be the first manifestation of Whipple's disease.

\section{Case Report}

\section{History}

A 36-year-old African American male presented to the emergency department with a one-day history of sudden decreased vision, redness, and pain with eye movement of the left eye. Two months prior, he had been treated for conjunctivitis in the left eye which had resolved. He had a history of untreated hypertension. His social history was positive for tobacco use and employment within the last year as a sewer worker. There was no recent history of travel or trauma. Reviews of systems were negative for abdominal symptoms, headache, weight loss, and arthritis.

Best-corrected visual acuities were 20/10 in the right eye and count fingers at 2 feet in the left eye and no improvement with pinhole. There was a grade 1 afferent pupillary defect on the left eye. Ocular motility and confrontation visual fields were normal in both eyes. Intraocular pressure was $17 \mathrm{mmHg}$ in both eyes via Goldmann tonometry. Right eye anterior segment examination was unremarkable. The left eye had diffuse hyperemia and grade 2+ injection with circumlimbal flush, grade $4+$ cells and $2+$ flare and a $0.5 \mathrm{~mm}$ hypopyon. Posterior synechiae were presented at seven and ten o'clock. Dilated fundus examination was normal on the right. Left eye revealed grade $2+$ vitreous cells, mild disc edema and vascular sheathing with a frosted branch appearance involving the inferior temporal arcade. Intraretinal and preretinal hemorrhages occupied a twodisc diameter area of necrosis inferior to the optic nerve. Panuveitis OS was made with differential diagnosis of CMV retinitis, sarcoidosis, and TB were suspected. HIV, immune panel, RPR, MHA-TP, ACE, Lysozyme, HLA-B27, and CXR were ordered and admission for further evaluation was recommended; however, the patient declined and left against medical advice.

Nine weeks later, he returned with chief complaint of persistent poor vision on the left and a one-week history of headache with sudden loss of vision of the temporal field on the right. This was preceded by a witnessed black out spell with no shaking, abnormal movements, or incontinence. After awaking he experienced numbness and tingling over his whole right side accompanied by headache and visual loss. Eight weeks prior, he had noted wrist and elbow pain. Four weeks prior, he had trouble with short-term memory and developed paranoid ideation. Patient appeared oriented times three and no slurred speech. He admitted that he has been taking 800 mg Ibuprofen and Tylenol for headaches as needed.

\section{Diagnostic Data}

On examination, his vision was $20 / 20$ on the right with some eccentric viewing and 20/200 on the left. A grade 1 afferent pupillary defect was still present on the left. Color vision was $12 / 12$ OD, $1 / 12$ OS. Motility was full and unrestricted. A right homonymous hemianopia was found on confrontation field and confirmed full threshold visual field test (Figure 1).

Humphrey Visual Field 30-2 showed depression consistent with right homonymous hemianopsia on right eye and diffused depression on the left eye consistent with optic atrophy. Intraocular pressures were $20 \mathrm{mmHg}$ OD and $18 \mathrm{mmHg}$ OS. Anterior segment showed normal findings right eye and posterior synechiae at $6 \& 9$ o'clock in the left eye. Fundus examination revealed normal optic nerve and retina right eye but disc pallor, arteriolar sheathing and attenuation involving the inferior temporal arcade left eye (Figure 2a, 2b, \& 2c). 
Open Access Journal of Ophthalmology

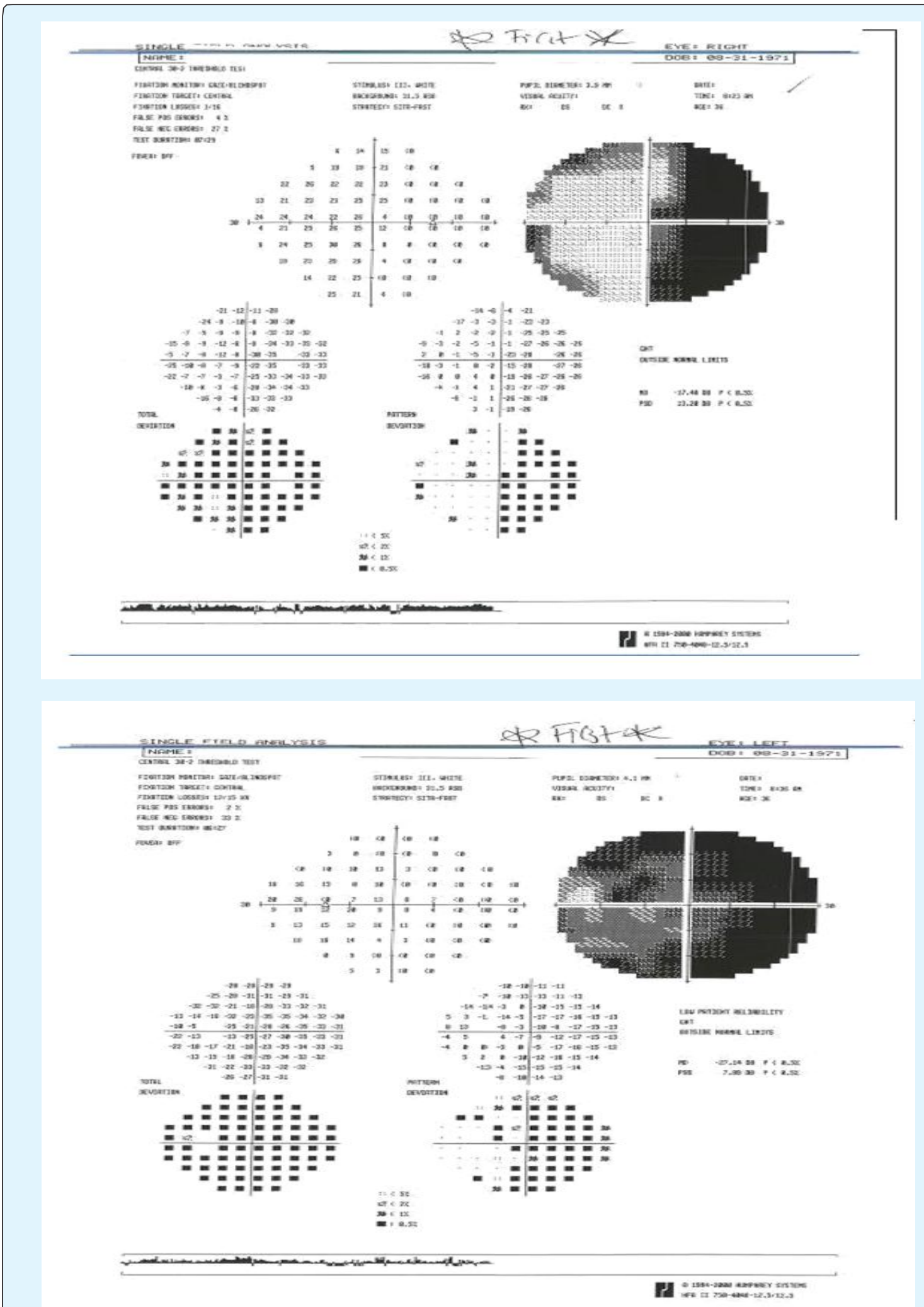

Figure 1: Humphrey Visual Field 30-2 showed depression consistent with right homonymous hemianopsia on right eye and diffused depression on the left eye consistent with optic atrophy. 


\section{Open Access Journal of Ophthalmology}

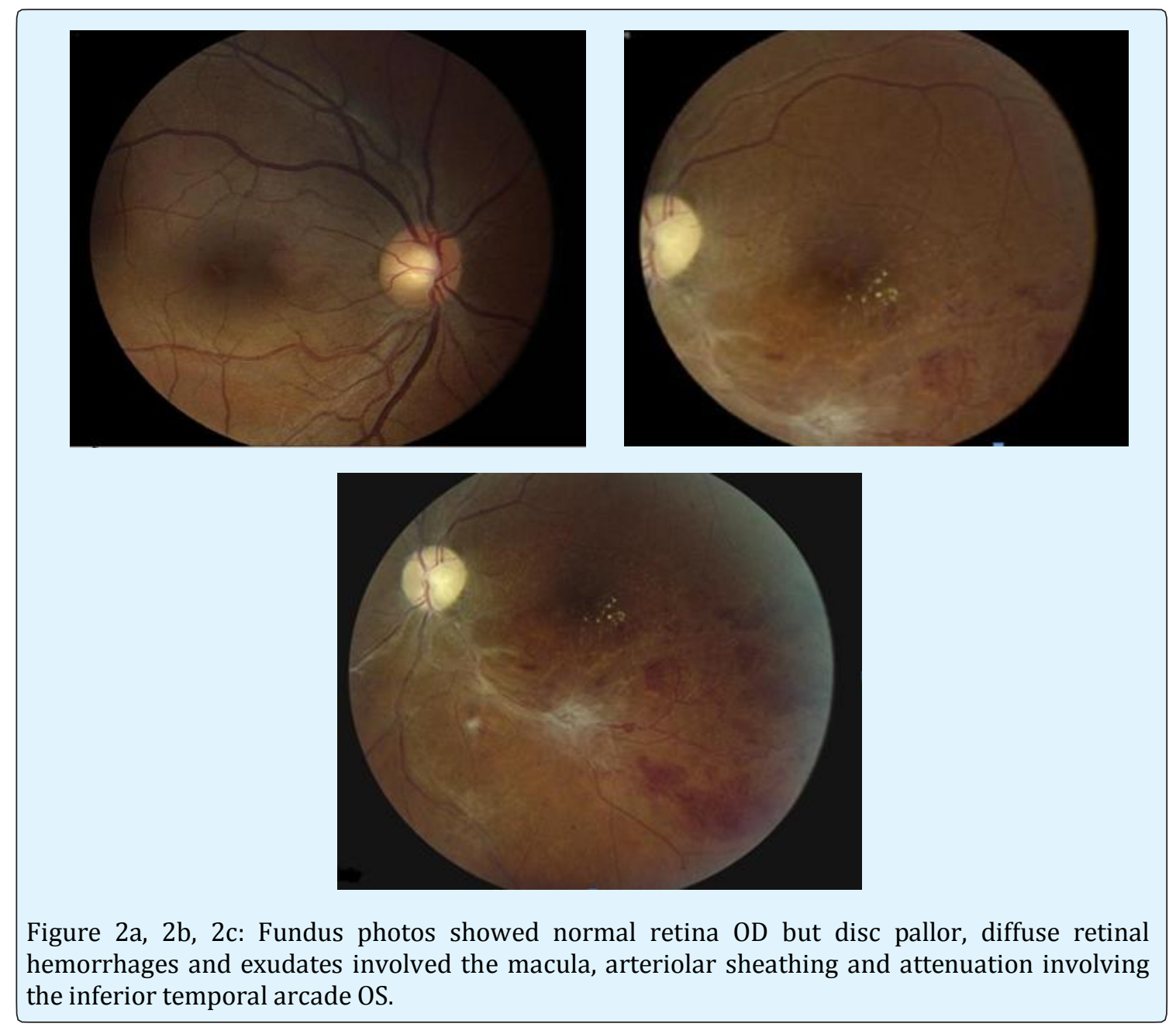

Diffuse retinal hemorrhages and exudates involved the macula and inferior temporal retina appeared less active than when originally seen 9 weeks prior. Neurologic examination uncovered a proximal weakness in the right leg. Differential diagnosis of chronic panuveitis left eye secondary to infections or immune-mediated (Table 1) and right temporal homonymous hemianopsia right eye with high suspicion for stroke secondary to uncontrolled hypertension and noncompliance to medication were suspected. Stat laboratory workups, CT scan, and neurology consult were ordered. Head CT scan found a ring enhancing mass with central hypodensity in the left basal ganglia. Sedimentation rate was $89 \mathrm{~mm} / \mathrm{hr}$. Antithrombin III and Lysosyme were slightly elevated at 148 and 23. ANA, HIV, HLA-B27, Lyme titers, RPR, RF, CXR, and PPD were negative. Differential diagnosis at this point is: metastic or neoplastic disease. ANCA, Bartonella, Brucella, CMV IgG/IgM, Cryptococcus, Coccidioides, MHATP, Toxoplasmosis IgG/IgM, West Nile IgG/IgM, Toxocara antibody, and urine Histoplasmosis were ordered and results were negative (Table 2).

\begin{tabular}{|c|c|c|c|}
\hline Infection & Immune-mediated & $\begin{array}{c}\text { Metastic/Neoplastic } \\
\text { Masquerader }\end{array}$ & Syndromes confined primarily to the eye \\
\hline Lyme disease & Sarcoidosis & Leukemia & Sympathetic ophthalmia \\
\hline HIV/CMV & Behcet's & Lymphoma & Leber's neuroretinitis \\
\hline Herpes virus & Ankylosing spondylitis & Syphilis & Acute retinal necrosis \\
\hline Toxoplasmosis & Reactive arthritis & & \\
\hline
\end{tabular}




\section{Open Access Journal of Ophthalmology}

\begin{tabular}{|c|c|l|l|}
\hline Leprosy & $\begin{array}{c}\text { Systemic lupus } \\
\text { erythematosus }\end{array}$ & & \\
\hline Tuberculosis & Vogt-Koyanagi-Harada & & \\
\hline Whipple's & & & \\
\hline West Nile & & & \\
\hline Bartonella & & & \\
\hline
\end{tabular}

Table 1: Differential diagnose of panuveitis/chronic Vasculitis.

\begin{tabular}{|c|c|}
\hline Test & Result \\
\hline ESR & $89(\mathrm{H})$ \\
\hline RPR & Negative \\
\hline ACE & Negative \\
\hline ANA & Negative \\
\hline $\mathrm{RF}$ & Negative \\
\hline HLA-B27 & Negative \\
\hline Antithrombin III & $148(\mathrm{H})$ \\
\hline MHA-TP & Negative \\
\hline Lyzosyme & $23(\mathrm{H})$ \\
\hline C-Anca & Negative \\
\hline HIV & Negative \\
\hline Lyme titer & Negative \\
\hline Brucella & Negative \\
\hline Bartonella & Negative \\
\hline Cryptococcus & Negative \\
\hline Coccidioides & Negative \\
\hline CXR & Negative \\
\hline VZV & Negative \\
\hline Toxoplasmosis IgG/IgM & Negative \\
\hline West Nile IgG/IgM & Negative \\
\hline Urine Histoplasmosis & Negative \\
\hline Toxocara antibody & Negative \\
\hline Cardioechogram & Negative \\
\hline Esophagogastro- duodenalscopy biopsy & Negative T.whipplei \\
\hline CT of chest, abdomen, \& pelvis & Negative lesion \\
\hline
\end{tabular}

\begin{tabular}{|c|l|}
\hline CSF studies & Results \\
\hline T. whipplei DNA PCR & Negative \\
\hline Toxo PCR & Negative \\
\hline Gram stain & Negative \\
\hline HSV/VZV & Negative \\
\hline Crypto & Negative \\
\hline M. Tub & Negative \\
\hline India Ink & Negative \\
\hline
\end{tabular}

Table 2: Laboratory tests.

Brain MRI (Figure 3) revealed left thalamic/left medial temporal lobe peripherally enhancing mass/hemorrhage with surrounding T2/FLAIR signal with similar lesion seen in the left posterior parietal lobe. The findings were 


\section{Open Access Journal of Ophthalmology}

"consistent with a possible metastatic lesion or toxoplasmosis given the proper clinical setting." Chest, abdomen, and pelvis CT found no evidence of malignancy. Spinal fluid examination revealed 41 WBC's, normal protein, negative gram Cryptococcal, and Indian ink stain.
HSV/VZV, mycobacterium tuberculosis, Toxoplasmosis and T. Whipplei spinal fluid PCR were negative. Esophagogastroduodenoscopy (EGD) with biopsy was negative for T. Whipplei.

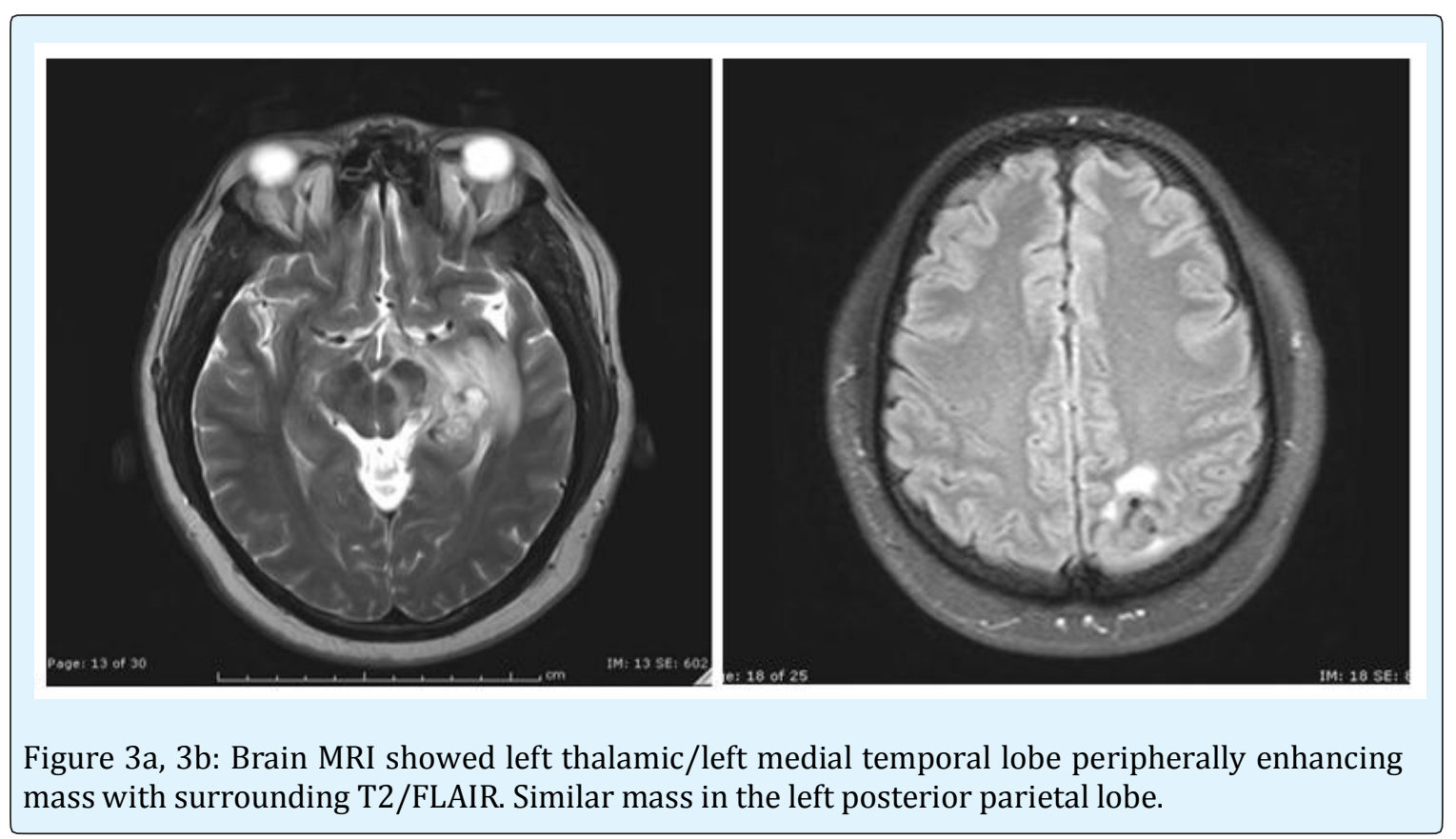

\section{Diagnosis}

A brain biopsy was performed which revealed foci of reactive changes, combined with the presence of intracytoplasmic periodic acid-Schiff reagent (PAS)- positive organisms within macrophages (Figure 4) which were consistent with a diagnosis of cerebral Whipple's disease (Figure 5). Cultures were not performed.

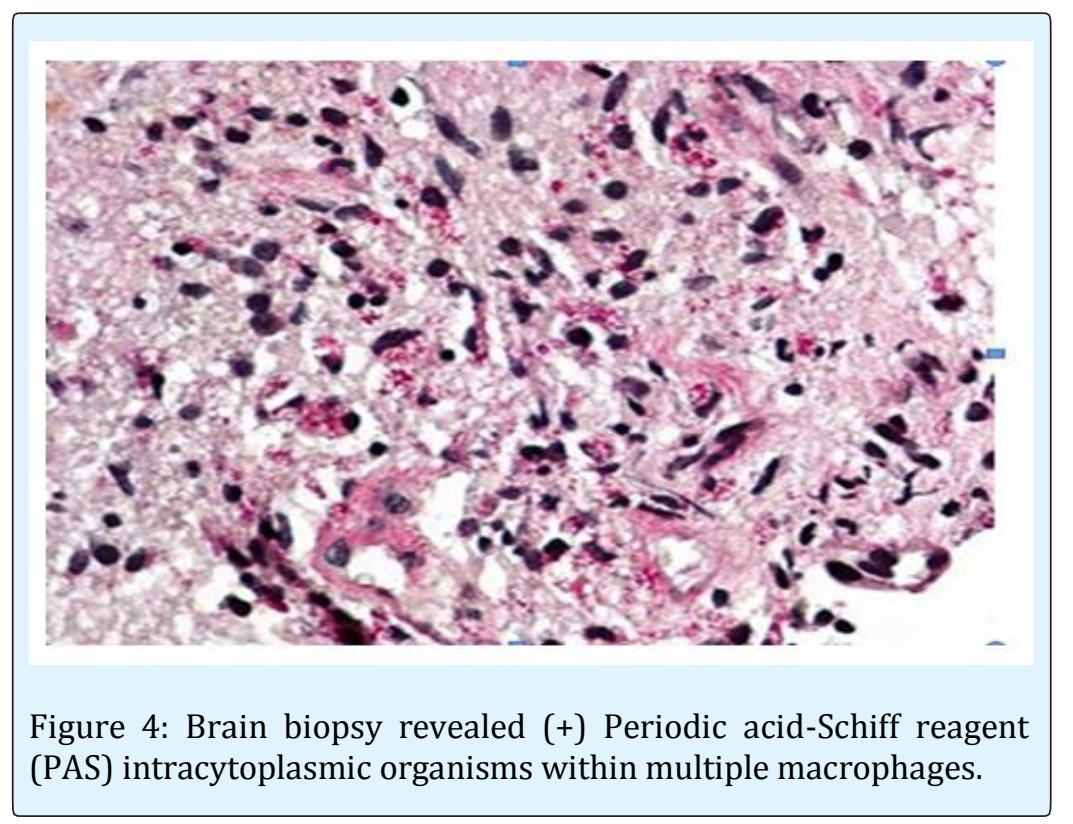

Thanh Le, et al. CNS Whipple's Disease Heralded by Retinal Vasculitis. J 


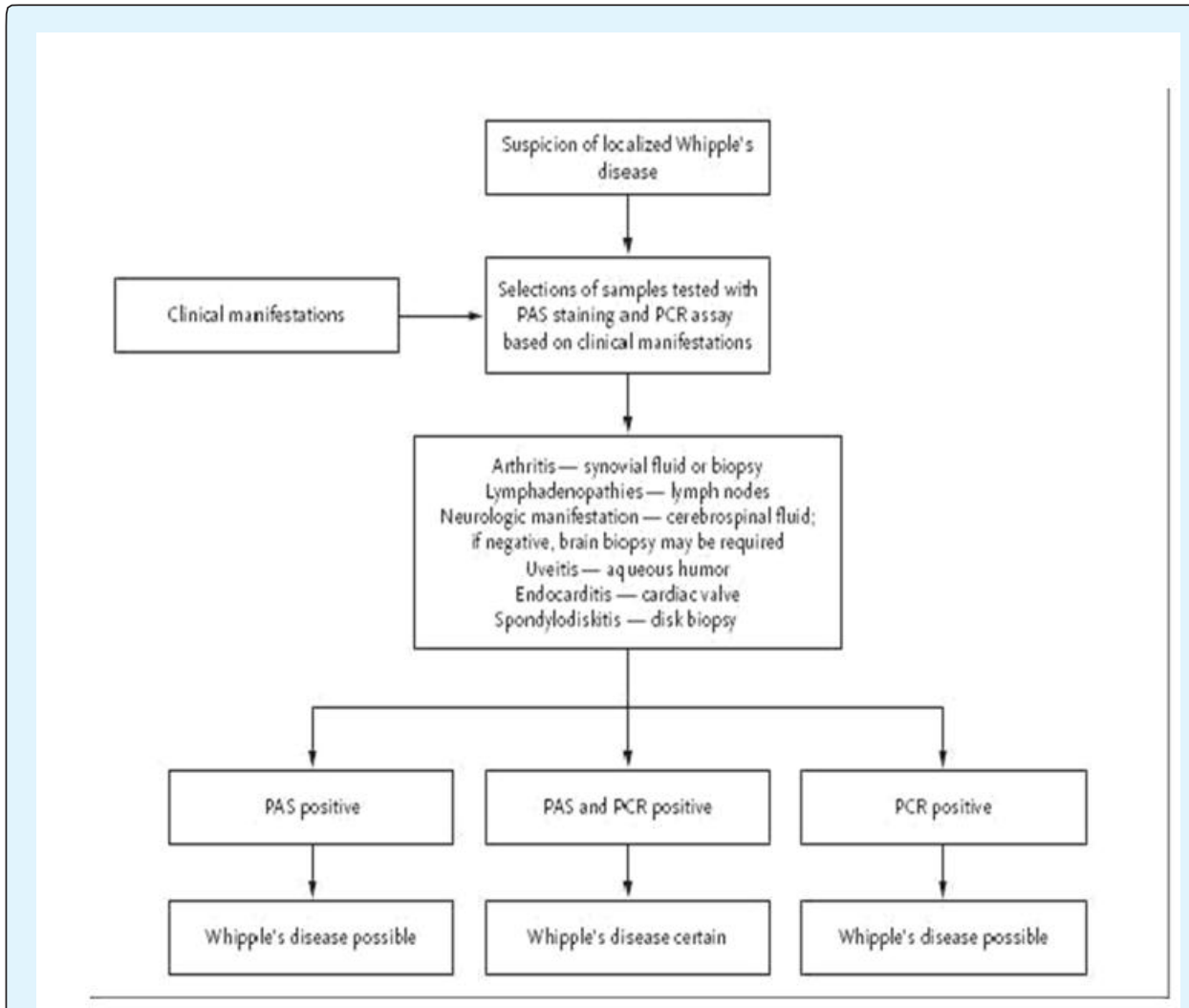

Figure 5: Step in diagnosis.

\section{Treatment and Follow-up}

The patient was treated with IV Rocephin for a total of four weeks followed by double strength Bactrim.
Repeated MRI scan a month after treatment (Figure 6).

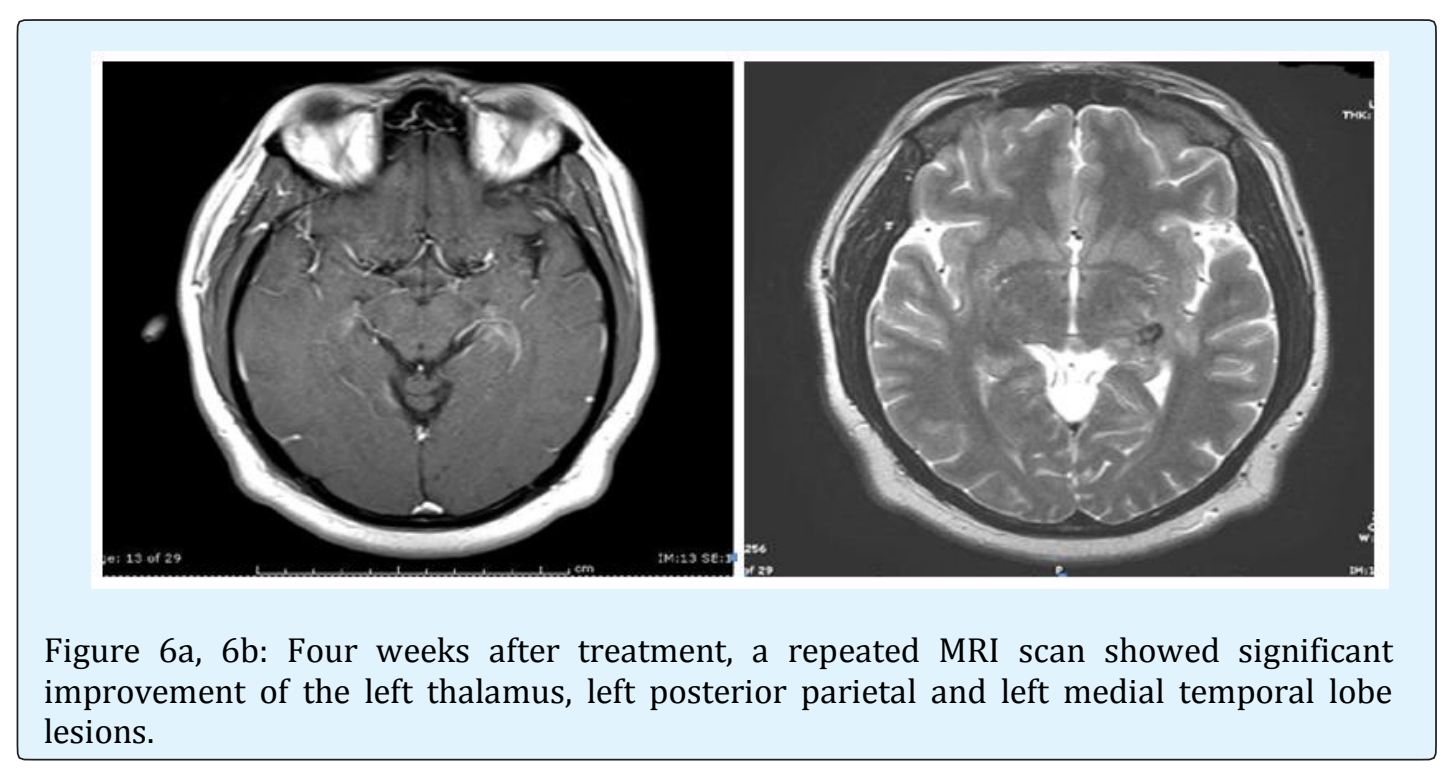




\section{Open Access Journal of Ophthalmology}

Showed significant improvement of the left thalamus, left posterior parietal and left medial temporal lobe lesions. At the same time, ophthalmologic examination found no change in visual acuity or ocular symptoms but new pre-retinal hemorrhages were noted (Figure 7a \& b).

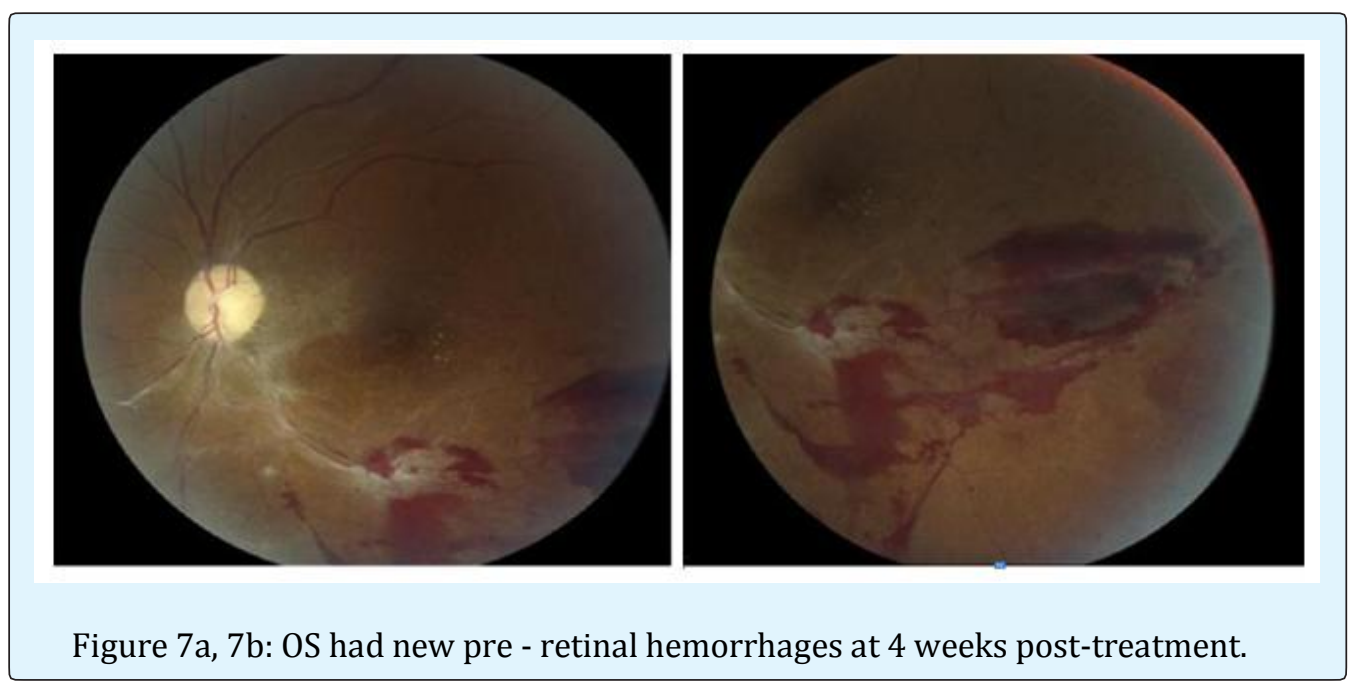

One month later, a right homonymous hemianopia OD and generalized depression OS remained on full threshold

visual field test but pre-retinal hemorrhage was improved (Figure 8 \& 9).

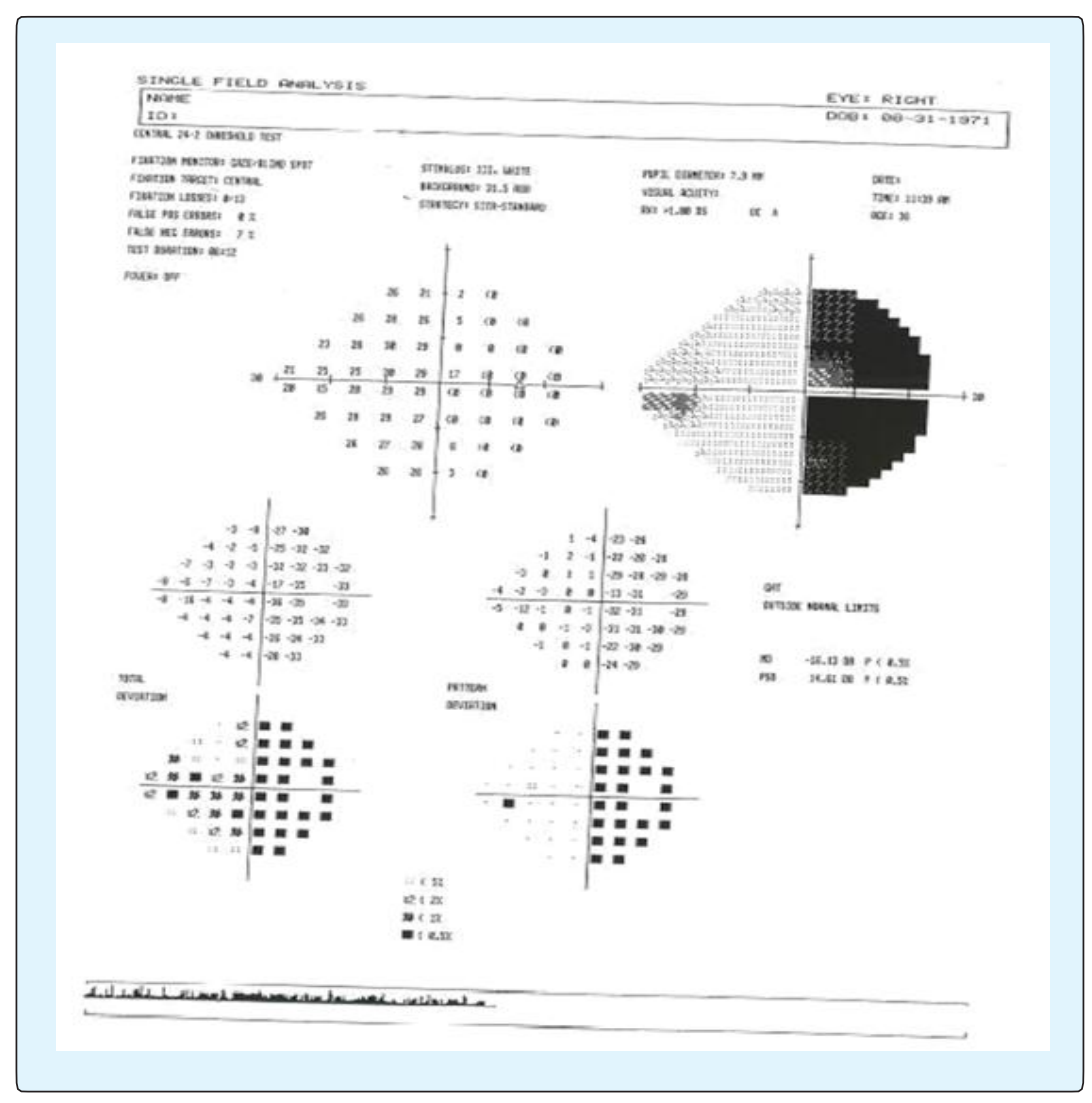




\section{Open Access Journal of Ophthalmology}
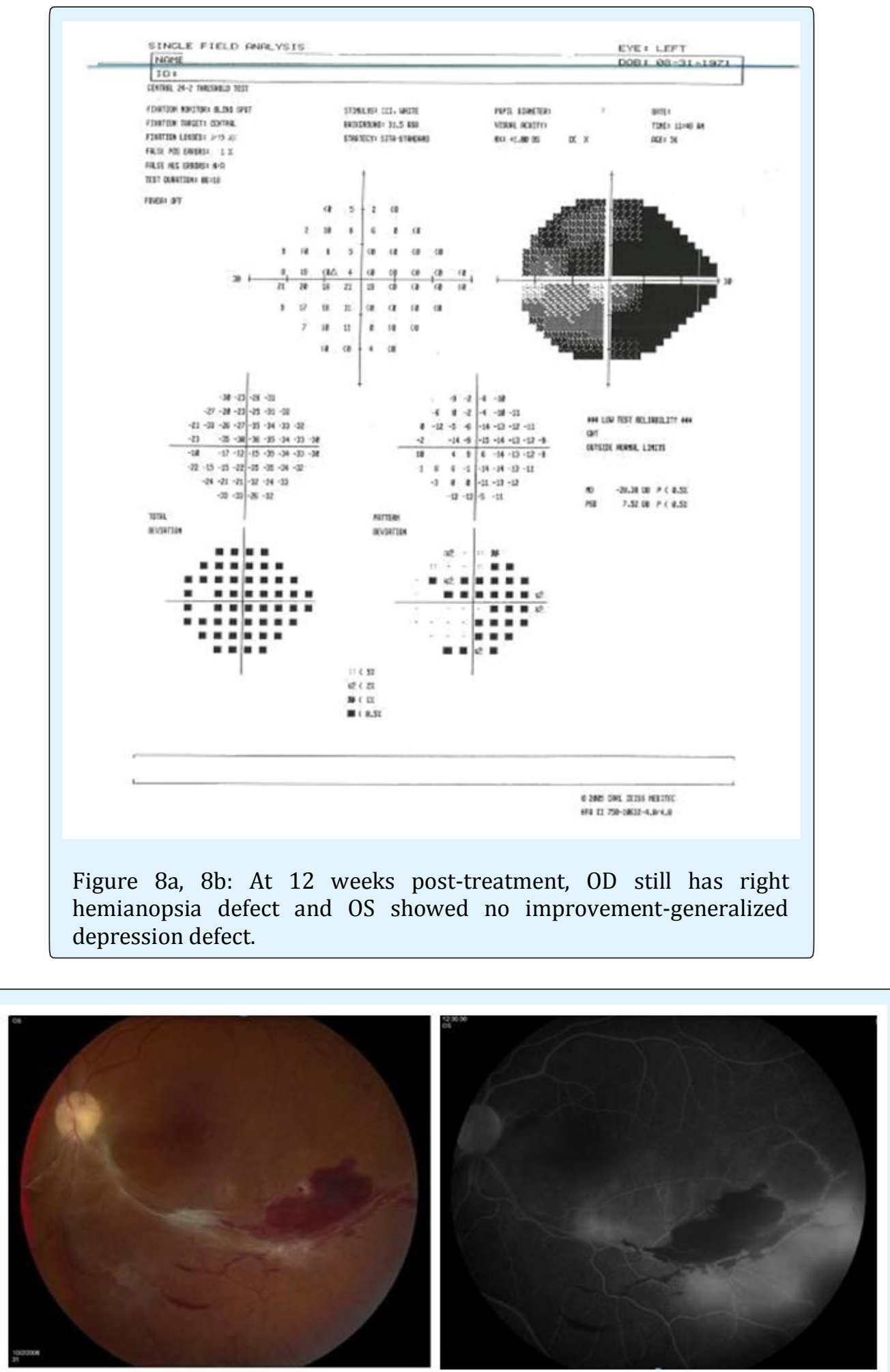

Figure 9a, 9b: At 12 week's post-treatment, pre-retinal hemorrhage OS improved. 


\section{Open Access Journal of Ophthalmology}

\section{Discussion}

Whipple's disease is a rare, chronic, multi-organ, bacterial infection that primarily involves the gastrointestinal tract and its lymphatic drainage in middle age men. The most common presenting manifestations of Whipple's disease are weight loss, diarrhea, migratory non-deforming seronegative polyarthralgias and abdominal pain. Extra intestinal involvement is found primarily in the central nervous system, lungs, and heart, although other sites including the eye may be affected. Ocular inflammation may occur alone or with gastrointestinal, neurologic, or other systemic manifestations (Table3).

\begin{tabular}{|c|c|c|}
\hline Ophthalmic (4) & Systemic & Neurologic \\
\hline Uveitis/Panuveitis,Vitritis & Diarrhea (80\%) & Supranuclear ophthalmoplegia \\
\hline Papilledema & Weightloss & Cognitive impairment \\
\hline $\begin{array}{c}\text { Retinal hemorrhage, cappillary } \\
\text { occlusion }\end{array}$ & $\begin{array}{c}\text { Migratory non-deforming seronegative } \\
\text { polyarthralgias (70\%) }\end{array}$ & $\begin{array}{c}\text { Oculomasticatory } \\
\text { myohythmia=Pathognomonic (4) }\end{array}$ \\
\hline Corneaulcer & Arthritis & Ataxia \\
\hline Chemosis, & Lymphadenopathies & Upper motor neuron disorders \\
\hline Glaucoma & Spondilodiskitis & Headaches \\
\hline Optic atrophy & Endocarditis & Dementia \\
\hline Epiphora & Fever?chill & Seizures \& somnolence \\
\hline Retinal Vasculitis & Increased skin pigmentation & Myoclonus \\
\hline Exudates/Hypopyon & Chronic nonproductive cough & \\
\hline Superficial punctate Keratitis & Pleural effusion/chest pain & \\
\hline
\end{tabular}

Table 3: Signs and symptoms of Whipple's.

The classic Whipple's disease affects the gastrointestinal tract causing diarrhea, weight loss, and migratory arthritis. Approximately $33 \%$ of patients presented with neurologic signs of cognitive impairment and supranuclear ophthalmoplegia [3]. Although less common, oculomasticatory myorhythmia is pathognomonic of the disease. Central nervous system (CNS) involvement can present in classic Whipple's in recurrence of the disease or in isolation [3]. Less common is the findings of cardiac and ophthalmic signs.

Roughly $15 \%$ of patients with Whipple's disease do not have the classic signs and symptoms of the disease. Members within this group can present with isolated neurologic, ocular or both findings without histologic evidence of intestinal involvement [3]. The most common neurologic findings are cognitive impairment, supranuclear ophthalmoplegia, ataxia, and upper motor neuron disorders. Oculomasticatory myorhythamia, (consisting of pendular mystagmus or smooth vergent mystagmus associated with tongue and mandibular myoclonus) although less common is pathognomonic for the disease [4].

Chan, et al, reported 77 cases of neurologic Whipple's with four involving the CNS only, 10 with CNS and ocular findings, and 21 cases of isolated ocular involvement [4]. Ophthalmic signs and symptoms can be the first manifestation of

Whipple's disease as described in the case presented here. Intraocular signs include: vasculitis with vitreous, hemorrhages, exudates, capillary occlusion, vitritis, panuveitis, and optic atrophy [4]. The presence of microorganisms in the retina of patient's with a diagnosis of Whipple's disease has been proven in several instances $[1,5]$. More recently the presence of T. whipplei in the retina and vitreous has been established by diagnostic polymerase chain reaction (PCR) [4].

The exact pathophysiology of Whipple's disease is unknown. There are predisposing factors such as male sex, positive HLA-B27, and occupational exposure to sewage material. One possibility is fecal oral transmission followed by invasion of tissues in those patients whose immune system is compromised in a manner yet to be defined. A proposed mechanism is due to defective macrophages given their abundance in infected tissues $[3,6]$. Recommended criteria for a definitive diagnosis of Whipple's disease are PAS staining of intracytoplasmic inclusions within macrophages and positive PCR for $T$. whipplei [3]. PAS staining is non-specific [3] and can be 


\section{Open Access Journal of Ophthalmology}

present in Mycobacerium avium and Histoplasmosis disease processes.

PCR may also be negative in denocarditis and CNS Whipple's disease [7-9].

In this case, the combination of PAS positive intracytoplasmic macrophage inclusions on brain biopsy, an otherwise negative workup, together with the ocular findings strongly suggested Whipple's disease. Marked improvements of the brain lesions and systemic symptoms with IV Ceftriaxone lead to a definitive diagnosis of Whipple's disease. Although brain lesions cleared and ocular symptoms improved, permanent optic atrophy and the visual defect remained (Figure 7). Ocular signs appeared to worsen slightly after one month of treatment. However, two months later, the vasculitis showed signs of resolution (Figure 8). It is possible that retinal involvement in this case was due to an immune hypersensitivity reaction without the actual presence of organisms in the retina $[5,10]$.

CNS involvement is a feared complication with a poor prognosis [11] and recurrences are common even after an initial response to antibiotics [1]. Re-infection is also a real concern in treated patients given that T. whipplei is common in the environment and these patients may have a predisposing immune defect $[5,11,12]$. Treatment for CNS and Ocular Whipple's disease remains unclear. Current recommendations include four weeks of IV ceftriaxone followed by 1-2 years of oral double strength Trimethoprim/Sulfamethoxazole (Bactrim) which was first proposed for its ability to penetrate the blood brain barrier [13]. But even with antibiotic treatment, CNS Whipple's tends to relapse.

\section{Conclusion}

In summary, we presented a patient who initially sought medical attention for ocular complaints and was later found to have CNS Whipple's disease diagnosed by a brain biopsy. He was treated with IV ceftriaxone and oral Trimethoprim/Sulfamethoxazole with improvement of both CNS and systemic symptoms. Although rare, Whipple's disease should be considered in any unexplained chronic uveitis especially if accompanied by CNS, gastro-intestinal, or migratory polyarthralgia sysmptoms.

\section{Reference}

1. Knox DL, Green WR, Troncoso JC, Yardley JH, Hau J et al. (1995) Cerebral ocular Whipple's disease: A 62year oodyssey from death to diagnosis. Neurology 45 : 617-625.

2. Rickman LS, Freeman WR, Green WR, Feldman ST, Sullivan J, et al. (1995) Uveitis caused by Tropheryma whippelli (Whipple's bacillus) [brief report]. N Engl J med 332 (6): 363-66.

3. Fenollar F, Puechal X, Raoult D (2007) Whipple's Disease. N Engl J Med 356(1): 55-66.

4. RY Chan, LA Yannuzzi, CS Foster (2001) Ocular Whipple's Disease: earlier Definitive Diagnosis. Ophthalmology 108(12): 2225-2231

5. Font RL, Rao NA, Issarescu S, William J, McEntee (1978) Ocular involvement in Whipple's disease: light and electron microscopic observeations. Arch Ophthalmol 96(8): 1431-1436.

6. Desnues B, Ihrig M, Raoult D, J L Mege (2006) Whipple's Disease: a macrophage disease. Clin Vaccine Immunol 13(2): 170-178

7. Williams OM, Nighttinglae AK, Hartley J (2007) Letter to the Editor. Whipple's Disease. N Engl J Med 356(14): 1479-1480

8. Bramkamp M, Ruggieri F, Schneermann M (2007) Letter to the Editor. Whipple's Disease. N Engl J Med 356: 1480-1481.

9. Wilson IG (1997) Inhibition and facilitation of nucleic acid amplification. Appl Environ Microbiol 63(10): 3741-3751.

10. Bavila MP, Jalkh AE, Feldman E, Trempe CL, Schepens CL (1984) Manifestations of Whipple's Disease in the Posterior Segment of the Eye. Arch Ophthalmol 102(3): 384-390.

11. Schnider PJ, Reisinger EC, Gerschlage W, Müller C, Berger T, et al. (1996) Long-term follow-up in cerebral Whipple's disease. Eur J Gastroenterol Hepatol 8(9): 899-903. 


\section{Open Access Journal of Ophthalmology}

12. Zinkernagel AS, Gmur R, Fenner L, Schaffner A, Schoedon G, et al. (2003) Marginal and subgingival plaque-a natural habitat of tropheryma whippelii?. Infection 31(2): 86-91.

13. Ryser RJ, Locksley RM, Eng Sc, Dobbins WO, Schoenknecht FD, et al. (1984) Reversal of dementia associated with Whipple's disease by Trimethorpimsulfamethoxazole, drugs that penetrate the bloodbrain barrier. Gastoenterology 86(4): 745-52. 